\title{
Global MicroRNA Expression Profiling Identifies Unique MicroRNA Pattern of Radioresistant Glioblastoma Cells
}

\author{
JAKUB ONDRACEK ${ }^{1 *}$, PAVEL FADRUS ${ }^{2 *}$, JIRI SANA $^{1,3}$, ANDREJ BESSE ${ }^{1}$, TOMAS LOJA ${ }^{1}$, MAREK VECERA ${ }^{1}$, \\ LENKA RADOVA $^{1}$, MARTIN SMRCKA $^{2}$, PAVEL SLAMPA $^{4}$ and ONDREJ SLABY ${ }^{1,3}$ \\ ${ }^{1}$ Central European Institute of Technology (CEITEC), Masaryk University, Brno, Czech Republic; \\ ${ }^{2}$ Department of Neurosurgery, University Hospital Brno, Masaryk University, Brno, Czech Republic; \\ ${ }^{3}$ Department of Comprehensive Cancer Care, Masaryk Memorial Cancer Institute, \\ Masaryk University, Brno, Czech Republic; \\ ${ }^{4}$ Department of Radiation Oncology, Masaryk Memorial Cancer Institute, \\ Masaryk University, Brno, Czech Republic
}

\begin{abstract}
Glioblastoma multiforme (GBM) is the most aggressive intracranial tumor characterized with infaust prognosis. Despite advances in neurosurgical and radiotherapeutic techniques and chemotherapy, the median overall survival ranges between 12-15 months from diagnosis. The main cause of treatment failure is considered the presence of tumor cells resistant to conventional therapy, mainly radiotherapy. MicroRNAs (miRNAs) are small, non-coding RNAs that function as post-transcriptional regulators of gene expression and have been repeatedly proven to play important roles in pathogenesis and biological features of many cancers, including GBM and its radioresistant phenotype. In our study, we established radioresistant cells from the commonly used human GBM cell lines T98G, U87MG and U251. Consequently, we performed global miRNA expression profiling in both radioresistant and parental cell lines and identified 113 miRNAs with significantly different expression $(p<0.05)$ between these two groups (73 miRNAs were up-regulated, 40 miRNAs were down-regulated). Some of these miRNAs have been previously described in relation to ionizing radiation, and others were herein identified for the first time. We believe that after deeper functional investigation of identified miRNAs in relation to radioresistance, these miRNAs present potential predictive biomarkers or therapeutic targets in GBM.
\end{abstract}

*These Authors contributed equally to this study.

Correspondence to: Assoc. Professor Ondrej Slaby, Ph.D. Masaryk University, Central European Institute of Technology (CEITEC), Molecular Oncology II, University Campus Bohunice, Building A35, Kamenice 5, 62500 Brno, Czech Republic. Tel: +420 549497574, Fax: +420 549497574, e-mail: on.slaby@gmail.com

Key Words: Glioblastoma, radioresistance, microRNA.
Glioblastoma multiforme (GBM) is a highly aggressive primary brain tumor of astrocytic origin accounting for nearly $50 \%$ of all gliomas with incidence of 3-4 new cases per 100,000 individuals $(1,2)$. Although metastases are unusual, GBM is characterized by infiltrative growth and early recurrences. Current standard therapeutic protocol consists of maximal surgical resection followed by concomitant chemoradiotherapy with temozolomide and adjuvant temozolomide in monotherapy. Unfortunately, GBM is often chemo-radioresistant and thus, adjuvant therapy fails. The prognosis of GBM patients is very poor with median overall survival ranging between 12 and 15 months from diagnosis $(1,2)$. A lot of effort has been spent in researching of both suitable biomarkers that could predict response to the therapy as well as drugs and therapeutic targets that could overcome GBM resistance. Over the past decade, involvement and functional roles of microRNAs (miRNAs) in GBM radioresistance were described $(3,4)$. MiRNAs are short, approximately 18-25 nucleotides long, non-coding single-stranded RNAs with an ability to posttranscriptionally regulate gene expression. Due to this feature, miRNAs are able to regulate multiple cellular processes, either physiological or pathological, such as apoptosis, proliferation, cell cycle, angiogenesis, differentiation. A number of miRNAs have been also identified to be deregulated in various cancers including GBM and some of them were associated with clinical outcomes and radioresistance (5-7). However, involvement of miRNAs in the molecular processes of cell response to the ionizing radiation in glioma is still poor understood. Therefore, we established radioresistant GBM cell lines in which we have subsequently performed global miRNA expression profiling in order to identify miRNAs deregulated in these cells and associated with resistance to the ionizing radiation in GBM. We believe that after deeper investigation 
of functional roles of these miRNAs in radioresistance in vitro, they present potential predictive biomarkers or therapeutic targets in GBM.

\section{Materials and Methods}

GBM cell lines and establishment of radioresistant cells. GMB cell lines U251, T98G, and U87MG as well as their radioresistant counterparts were cultivated in DMEM medium enhanced by $10 \%$ heat-inactivated fetal bovine serum, $100 \mu \mathrm{g} / \mathrm{ml}$ streptomycin and $100 \mathrm{U} / \mathrm{ml}$ penicillin, $4.5 \mathrm{~g} / 1 \mathrm{D}$-glucose, $2 \mathrm{mM}$ L-glutamine, $1 \%$ nonessential amino acids, and $10 \mathrm{mg} / 1$ sodium pyruvate. Cells were cultivated on $100-\mathrm{mm}$ culture dishes at $37^{\circ} \mathrm{C}$ in humidified atmosphere containing $5 \% \mathrm{CO}_{2}$.

All three GBM cell lines were firstly grown to approximately $60 \%$ confluence and irradiated with 2 Gy (Cs-137 $\gamma$-radiation, 2 Gy/min). When cells reached about $90 \%$ confluence, they were subcultured into new dishes. The cells were treated again with $2 \mathrm{~Gy}$ when they reached $60 \%$ confluence. This procedure was repeated until total dose was 32 Gy. After that, cells were irradiated by the same process but dose 4 Gy was used instead of $2 \mathrm{~Gy}$. This procedure was repeated until total dose of ionizing radiation was $60 \mathrm{~Gy}$. Parental cells were cultured under the same conditions without ionizing radiation treatment Multiply irradiated cells were named with suffix -R (Radioresistant), giving U251-R, T98G-R, U87MG-R. Parental cells were named with suffix -C (Control). For all assays, there was at least a four-week interval between the last fractionated irradiation and the experiment.

Colony formation assay. Diluted cell suspension was seeded into 6well plates, incubated overnight, irradiated by dose $4 \mathrm{~Gy}$, and cultured until cells form colonies. Subsequently, cells were fixed in $4 \%$ paraformaldehyde $(30 \mathrm{~min} / \mathrm{RT})$ and visualized by $0.5 \%$ crystal violet $(20 \mathrm{~min} / \mathrm{RT})$. The colonies were counted using GelCount (Oxford, USA). Calculation of surviving fractions (SF) of cells after irradiation was performed using the equation $\mathrm{SF}=$ colonies counted/(cells seeded*PE). Plating efficiency (PE) is the ratio of the number of colonies to the number of cells seeded, which were not treated by radiation (8). Experiments were repeated three times; each experiment was performed in triplicates. Statistical evaluations of data obtained from colony formation assay were performed on GraphPad Prism 5 software by a one-tailed non-parametric $t$-test.

MiRNA microarray analysis. Small RNA enriched total RNA was isolated using the Direct-zol RNA MiniPrep Kit (Zymo Research, USA). Nucleic acid concentrations and purities were controlled by UV spectrophotometry using Nanodrop ND-1000 (Thermo Scientific). To assess miRNA expression in radioresistant and control GBM cell lines, the samples were analyzed with Affymetrix GeneChip miRNA 3.0 arrays (Affymetrix, Santa Clara, CA, USA) containing 1733 probe sets for human mature miRNAs. Experiments were performed according to the Affymetrix standardized protocol for miRNA 3.0 arrays. Intensity values for each probe cell were calculated using Affymetrix GeneChip Command Console (AGCC). Quality control of the microarray was performed with the Affymetrix miRNA QC Tool, version 1.1.1.0.

Microarray expression data analysis. All data were pre-processed and further analyzed by the software packages included in the R/Bioconductor (9). Pre-processing was performed by the RMA method with default parameters as implemented in the Bioconductor package oligo (10). All data were log2-transformed. To identify differentially expressed miRNAs, the LIMMA approach (11) for paired samples was applied with additional Benjamini-Hochberg correction of $p$-values.

\section{Results}

Radioresistance analysis. Prior to miRNA expression analyses in the examined GBM cell lines, their resistance to ionizing radiation was evaluated. The colony formation assay has confirmed that all three GBM cell lines, which have received a total dose of $60 \mathrm{~Gy}$, form significantly higher number of colonies after 4 Gy irradiation in comparison to the parental control GBM cell lines (Figure 1). In concrete, cell lines U251-R, T98G-R, and U87MG-R form on average 3-times ( $p=0.0395$; Figure 1C), 3.5-times $(p=0.0363$; Figure $1 \mathrm{~F})$, and 1.6-times $(p=0.0277$; Figure 1I) more colonies than their parental cell lines U251-C, T98G-C, and U87MG-C, respectively.

Global miRNA expression analysis of radioresistant and parental GBM cell lines. Global miRNA expression analyses followed by statistical comparison of three pairwise radioresistant and parental GBM cell lines identified 113 significantly differently expressed miRNAs between these two groups $(p<0.05)$. From these, 73 miRNAs were upregulated and 40 miRNAs down-regulated in radioresistant cells; 21 miRNAs have shown the $p$-value less than 0.01 . The most up-regulated miRNA in radioresistant GBM cells was identified as miR-145 while the most down-regulated was miR-1271 (Table I).

\section{Discussion}

Radiotherapy plays an important role in oncological treatment of GBM patients. Nevertheless, this brain tumor is well known for its frequent resistance to ionizing radiation and, thus, adjuvant therapy often fails and GBM develop early recurrences. This results in a bad prognosis associated with short survival of GBM patients. In the presented study, we compared miRNA expression profiles in radioresistant and parental stable GBM cell lines U251, T98G, and U87MG. Our aim was to use global miRNA expression profiling and identify miRNAs that might be closely associated with radioresistance of GBM cells and, thus, could be promising predictive markers, as well as potential therapeutic targets in GBM patients.

Firstly, we established three GBM cell lines treated with total dose of $60 \mathrm{~Gy}$. Following evaluation of their resistance to the ionizing radiation confirmed significantly higher potential of these cell lines to form colonies after 4-Gy experimental treatment compared to the parental control cell cultures. Similar results were published by the Yang et al. who induced radioresistance in $\mathrm{U} 251 \mathrm{GBM}$ cell line by the fractionated ionizing radiation exposure in total dose $62 \mathrm{~Gy}$ (12). 

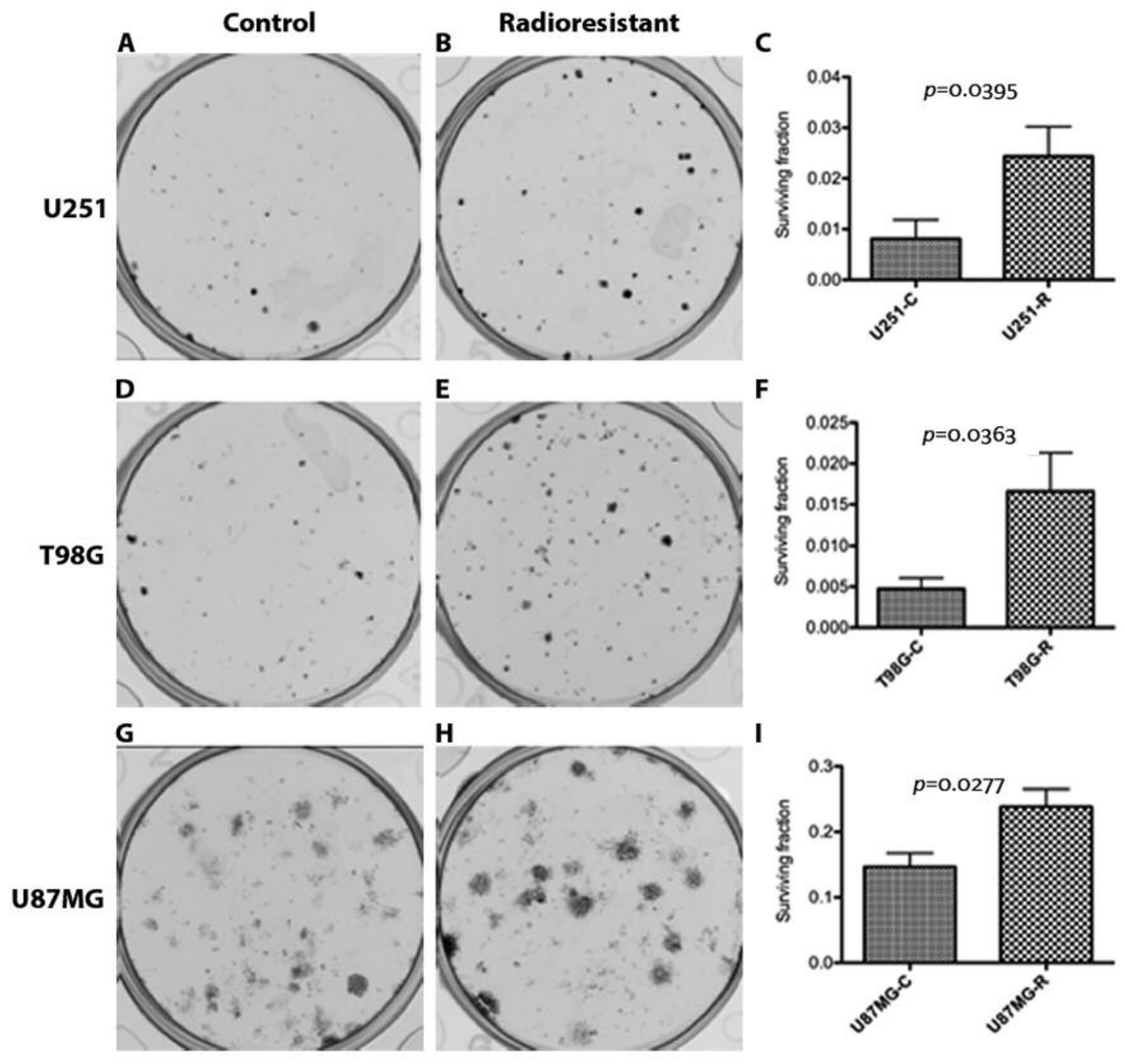

Figure 1. Colony formation ability of 4 Gy radiation treated $U 251-C / R(A, B), T 98 G-C / R(D, E)$ and $U 87 M G-C / R(G, H) G B M$ cell lines. Surviving fraction of (C) U251-C/R, (F) T98G-C/R and (I) U87MG-C/R GBM cell lines after 4 Gy radiation treatment (one-tailed non-parametric t-test).

The main aim of our work was to compare miRNA expression profiles in pairwise radioresistant and parental control GBM cell lines and identify miRNAs with significantly different expression levels between these groups. We identified 113 miRNAs with significantly different expression in radioresistant GBM cells $(p<0.05)$. Many of these miRNAs have been previously observed to be linked with radiation treatment, including miR-29, miR-218, miR145, miR-204, miR-126, miR-146b-5p, miR-31, miR-302a and miR-452 (13-22). Focusing on these miRNAs, several previous studies have been focused on the discovery of molecular mechanisms responsible for the tumor radioresistance caused by these miRNAs, mainly in oropharyngeal area. Lynam-Lennon et al. observed, similarly like in our study, that miR-31 is significantly down-regulated in isogenic model of radioresistance in oesophageal adenocarcinoma cells, both basally and in response to radiation. Ectopic re-expression of miR-31 led to expression alteration of 13 genes involved in DNA repair and significant re-sensitizing of radioresistant cells to radiation (13). Also miR-452 was one of four miRNAs, that have been identified by the comprehensive miRNA expression profiling to be differentially expressed in radioresistant laryngeal squamous cell carcinoma (14). Finally, in nasopharyngeal carcinoma cells, miR-204 is involved in radioresistance and epithelial-mesenchymal transition. Lu et al. described that both processes are regulated by the lncRNA NEAT1 through miR-204/ZEB 1 axis (15). Interestingly, the same processes 
Table I. MiRNAs with significantly different expression levels in radioresistant and parental control GBM cell lines $(p<0.05)$.

\begin{tabular}{|c|c|c|c|c|c|c|c|}
\hline miRNA & $\log \mathrm{FC}$ & AveExpr & $p$-Value & miRNA & $\log \mathrm{FC}$ & AveExpr & $p$-Value \\
\hline miR-663 & 0.98 & 7.21 & $<0.01$ & miR-4734 & 0.86 & 7.59 & 0.02 \\
\hline miR-10b-star & 1.64 & 3.61 & $<0.01$ & miR-4689 & 0.57 & 5.67 & 0.02 \\
\hline miR-4498 & 1.40 & 2.89 & $<0.01$ & miR-3178 & 0.86 & 8.30 & 0.03 \\
\hline $\operatorname{miR}-572$ & 1.05 & 1.99 & $<0.01$ & miR-4521 & -0.90 & 8.60 & 0.03 \\
\hline $\operatorname{miR}-4505$ & 0.95 & 5.63 & $<0.01$ & miR-146b-5p & 0.68 & 3.33 & 0.03 \\
\hline miR-4486 & 0.97 & 4.38 & $<0.01$ & miR-532-5p & -0.51 & 5.46 & 0.03 \\
\hline miR-4725-3p & 1.20 & 1.61 & $<0.01$ & miR-582-5p & -0.64 & 0.93 & 0.03 \\
\hline miR-1301 & 0.94 & 2.17 & $<0.01$ & $\operatorname{miR}-4251$ & -0.51 & 0.50 & 0.03 \\
\hline miR-10a-star & 1.33 & 2.29 & $<0.01$ & $\mathrm{miR}-4500$ & 0.70 & 1.36 & 0.03 \\
\hline miR-3185 & 1.07 & 6.78 & $<0.01$ & $\operatorname{miR}-4640-5 p$ & 0.78 & 3.41 & 0.03 \\
\hline miR-4707-5p & 0.88 & 6.96 & $<0.01$ & miR-3136-5p & -0.71 & 1.19 & 0.03 \\
\hline $\operatorname{miR}-29 b$ & -1.26 & 6.58 & $<0.01$ & miR-3940-5p & 0.53 & 8.41 & 0.03 \\
\hline miR-1908 & 0.81 & 7.98 & $<0.01$ & miR-150-star & 1.01 & 2.23 & 0.03 \\
\hline miR-218 & 0.80 & 2.63 & $<0.01$ & miR-518e & -0.53 & 0.69 & 0.03 \\
\hline miR-3065-5p & -1.52 & 3.73 & $<0.01$ & miR-212 & 0.92 & 1.28 & 0.03 \\
\hline miR-3910 & -0.79 & 1.49 & $<0.01$ & miR-143 & 2.19 & 2.43 & 0.03 \\
\hline let-7a-2-star & -0.78 & 4.46 & $<0.01$ & miR-2276 & -0.59 & 2.09 & 0.03 \\
\hline miR-145 & 3.23 & 2.72 & $<0.01$ & miR-31 & -0.51 & 7.08 & 0.03 \\
\hline miR-4497 & 1.01 & 10.03 & $<0.01$ & $\operatorname{miR}-548 c-5 p$ & -0.50 & 0.70 & 0.03 \\
\hline miR-4721 & 1.29 & 3.28 & $<0.01$ & miR-3621 & 0.62 & 5.39 & 0.03 \\
\hline miR-4697-3p & 1.20 & 1.39 & $<0.01$ & $\operatorname{miR}-4313$ & -0.53 & 1.25 & 0.03 \\
\hline miR-30b-star & 0.96 & 1.65 & 0.01 & miR-4430 & 0.77 & 3.06 & 0.03 \\
\hline miR-1231 & 1.23 & 4.68 & 0.01 & miR-422a & -0.73 & 6.10 & 0.04 \\
\hline miR-4778-5p & 1.07 & 1.65 & 0.01 & let-7e-star & 1.02 & 1.95 & 0.04 \\
\hline $\mathrm{miR}-378 \mathrm{e}$ & -0.84 & 2.61 & 0.01 & $\mathrm{miR}-4745-5 \mathrm{p}$ & 0.75 & 8.05 & 0.04 \\
\hline miR-4651 & 0.68 & 6.78 & 0.01 & miR-4466 & 0.56 & 9.55 & 0.04 \\
\hline $\operatorname{miR}-4440$ & -0.82 & 1.15 & 0.01 & $\operatorname{miR}-4518$ & -0.52 & 0.60 & 0.04 \\
\hline miR-204 & 1.26 & 3.15 & 0.01 & $\mathrm{miR}-520 \mathrm{~g}$ & -0.50 & 0.63 & 0.04 \\
\hline miR-217 & -0.65 & 0.95 & 0.01 & miR-4649-5p & 0.67 & 4.19 & 0.04 \\
\hline miR-1914-star & 0.76 & 1.80 & 0.01 & miR-185-star & -0.85 & 0.99 & 0.04 \\
\hline miR-126 & -0.70 & 3.89 & 0.01 & miR-302a & -0.53 & 0.82 & 0.04 \\
\hline miR-302d & 0.82 & 1.29 & 0.01 & miR-1909-star & 0.56 & 1.54 & 0.04 \\
\hline miR-224 & 0.70 & 5.15 & 0.01 & $\mathrm{miR}-4781-3 \mathrm{p}$ & -0.69 & 0.86 & 0.04 \\
\hline $\operatorname{miR}-2467-3 p$ & -1.05 & 1.18 & 0.02 & miR-138-2-star & 0.47 & 0.96 & 0.04 \\
\hline $\operatorname{miR}-431$ & 0.94 & 1.97 & 0.02 & miR-4439 & 0.49 & 0.99 & 0.04 \\
\hline $\mathrm{miR}-4750$ & 1.28 & 3.85 & 0.02 & miR-648 & -0.45 & 0.94 & 0.04 \\
\hline $\operatorname{miR}-4783-3 p$ & 0.63 & 2.65 & 0.02 & $\operatorname{miR}-452$ & 0.74 & 4.00 & 0.04 \\
\hline $\operatorname{miR}-1271$ & -2.86 & 2.23 & 0.02 & $\operatorname{miR}-570$ & -0.90 & 0.86 & 0.04 \\
\hline miR-664-star & 1.06 & 1.27 & 0.02 & miR-3682-3p & 0.78 & 1.74 & 0.04 \\
\hline miR-935 & 1.08 & 3.23 & 0.02 & miR-4462 & 0.73 & 1.54 & 0.04 \\
\hline miR-22 & -0.67 & 10.29 & 0.02 & miR-887 & 0.66 & 1.49 & 0.04 \\
\hline $\operatorname{miR}-4443$ & 0.85 & 5.14 & 0.02 & miR-331-3p & 0.84 & 3.60 & 0.04 \\
\hline miR-3197 & 0.91 & 2.64 & 0.02 & miR-767-3p & -0.57 & 1.21 & 0.04 \\
\hline miR-617 & -0.61 & 0.83 & 0.02 & miR-3679-5p & 0.48 & 4.00 & 0.04 \\
\hline miR-4634 & 1.25 & 3.96 & 0.02 & miR-4749-5p & 0.80 & 4.38 & 0.04 \\
\hline miR-3619-5p & 0.73 & 3.82 & 0.02 & miR-744-star & 0.47 & 1.30 & 0.05 \\
\hline miR-4324 & 0.91 & 2.14 & 0.02 & miR-4726-5p & -0.73 & 1.58 & 0.05 \\
\hline miR-1323 & -0.91 & 1.36 & 0.02 & miR-3188 & 1.13 & 3.28 & 0.05 \\
\hline miR-4682 & 0.64 & 1.13 & 0.02 & miR-4746-3p & 0.49 & 0.81 & 0.05 \\
\hline $\mathrm{miR}-378 \mathrm{f}$ & -0.76 & 5.59 & 0.02 & miR-759 & -0.52 & 0.56 & 0.05 \\
\hline miR-186-star & -0.72 & 0.94 & 0.02 & miR-3591-3p & -0.62 & 0.78 & 0.05 \\
\hline miR-199a-5p & 0.59 & 3.26 & 0.02 & $\operatorname{miR}-615-3 p$ & 0.47 & 2.42 & 0.05 \\
\hline miR-4741 & 0.77 & 6.11 & 0.02 & miR-762 & 0.48 & 8.48 & 0.05 \\
\hline $\operatorname{miR}-3617$ & 1.61 & 1.79 & 0.02 & miR-4454 & -0.50 & 11.04 & 0.05 \\
\hline miR-4507 & 0.55 & 5.79 & 0.02 & miR-652 & 0.54 & 4.56 & 0.05 \\
\hline miR-1290 & -1.27 & 5.83 & 0.02 & miR-3187-3p & 0.83 & 3.25 & 0.05 \\
\hline miR-378 & -0.58 & 6.43 & 0.02 & & & & \\
\hline
\end{tabular}

FC, Fold Change; AveExpr, average expression (signal on Affymetrix GeneChip). 
are regulated by the double-negative feedback loop between lncRNA TUG1 and miR-145 in bladder cancer cells (16). MiR-145 plays a crucial role in modulation of radiosensitivity also in prostate cancer and $\mathrm{HR}-\mathrm{HPV}^{+}$cervical cancer where another lncRNA MALAT1 acts as sponge for miR-145 and mediates its functioning $(17,18)$. In addition to miR-145, sensitivity of cervical cancer to the ionizing radiation is affected also by miR-218 via promoting radiation-induced apoptosis (19). Yang et al. have described that lincRNA-p21, regulator of cell proliferation, apoptosis and DNA damage response, negatively regulated the expression and activity of $\beta$-catenin in glioma stem cells (GSCs) and down-regulation of lincRNA-p21 resulted from up-regulation of $\mathrm{Hu}$ antigen $\mathrm{R}$ (HuR) expression caused by miR-146b-5p down-regulation. Expectedly, miR-146b-5p overexpression increased apoptosis and radiosensitivity, decreased cell viability, neurosphere formation capacity and stem cell marker expression, and induced differentiation in GSCs (20). In breast cancer, miR302a was down-regulated in irradiated cells. Additionally, the expression levels of miR-302a were inversely correlated with those of AKT1 and RAD52, two critical regulators of radioresistance. More promisingly, miR-302a sensitized radioresistant breast cancer cells to radiation therapy in vitro and in vivo and reduced the expression of AKT1 and RAD52 (21). Finally, miR-29 and miR-126 were both identified to be significantly down-regulated in the serum of the lung cancer patients after radiation treatment (22).

Altogether, our study revealed miRNAs differentially expressed in radioresistant GBM cell lines that may play a role in the molecular process of tumor cell response to radiotherapy in GBM patients.

\section{Conflicts of Interest}

The Authors have no conflict of interest.

\section{Acknowledgements}

This work was financially supported by the Czech Ministry of Health grants no. 15-34553A and 15-33158A; by the project MZ CR - RVO (MOU, 00209805); and by the Ministry of Education, Youth and Sports of the Czech Republic under the project CEITEC 2020 (LQ1601).

\section{References}

1 Stupp R, Mason WP, van den Bent MJ, Weller M, Fisher B, Taphoorn MJ, Belanger K, Brandes AA, Marosi C, Bogdahn U, Curschmann J, Janzer RC, Ludwin SK, Gorlia T, Allgeier A, Lacombe D, Cairncross JG, Eisenhauer E, Mirimanoff RO; European Organisation for Research and Treatment of Cancer Brain Tumor and Radiotherapy Groups.; National Cancer Institute of Canada Clinical Trials Group: Radiotherapy plus concomitant and adjuvant temozolomide for glioblastoma. N Engl J Med 352: 987-996, 2005.
2 Louis DN, Ohgaki H, Wiestler OD, Cavenee WK, Burger PC, Jouvet A, Scheithauer BW and Kleihues P: The 2007 WHO classification of tumours of the central nervous system. Acta Neuropathol (Berl) 114: 97-109, 2007.

3 Besse A, Sana J, Lakomy R, Kren L, Fadrus P, Smrcka M, Hermanova M, Jancalek R, Reguli S, Lipina R, Svoboda M, Slampa P and Slaby O: MiR-338-5p sensitizes glioblastoma cells to radiation through regulation of genes involved in DNA damage response. Tumour Biol 37: 7719-7727, 2016.

4 Besse A, Sana J, Fadrus P and Slaby O: MicroRNAs involved in chemo- and radioresistance of high-grade gliomas. Tumour Biol 34: 1969-1978, 2013.

5 Sana J, Radova L, Lakomy R, Kren L, Fadrus P, Smrcka M, Besse A, Nekvindova J, Hermanova M, Jancalek R, Svoboda M, Hajduch M, Slampa P, Vyzula R and Slaby O: Risk Score based on microRNA expression signature is independent prognostic classifier of glioblastoma patients. Carcinogenesis 35: 2756-2762, 2014.

6 Lakomy R, Sana J, Hankeova S, Fadrus P, Kren L, Lzicarova E, Svoboda M, Dolezelova H, Smrcka M, Vyzula R, Michalek J, Hajduch M and Slaby O: MiR-195, miR-196b, miR-181c, miR-21 expression levels and O-6-methylguanine-DNA methyltransferase methylation status are associated with clinical outcome in glioblastoma patients. Cancer Sci 102: 2186-2190, 2011.

7 Sana J, Hajduch M, Michalek J and Slaby O: MicroRNAs and glioblastoma: roles in core signalling pathways and potential clinical implications. J Cell Mol Med 15: 1636-1644, 2011.

8 Franken NAP, Rodermond HM, Stap J, Haveman J and van Bree C: Clonogenic assay of cells in vitro. Nat Protoc 1: 2315-2319, 2006.

9 Team RC: R: A Language and Environment for Statistical Computing. Foundation for Statistical Computing, Vienna, Austria 2013; http://www.R-project.org/.

10 Carvalho BS and Irizarry R: A framework for oligonucleotide microarray preprocessing. Bioinforma Oxf Engl 26: 2363-2367, 2010.

11 Smyth G: Limma: linear models for microarray data. In Gentleman R, Carey V, Dudoit S et al: Bioinformatics and Computational Biology Solutions Using R and Bioconductor. Springer, New York, 397-420, 2005.

12 Yang J-A, Liu B-H, Shao L-M, Guo ZT, Yang Q, Wu LQ, Ji BW, Zhu XN, Zhang SQ, Li CJ and Chen QX: LRIG1 enhances the radiosensitivity of radioresistant human glioblastoma U251 cells via attenuation of the EGFR/Akt signaling pathway. Int J Clin Exp Pathol 8: 3580-8590, 2015.

13 Lynam-Lennon N, Reynolds JV, Marignol L, Sheils OM, Pidgeon GP and Maher SG: MicroRNA-31 modulates tumour sensitivity to radiation in oesophageal adenocarcinoma. J Mol Med 90: 1449-1458, 2012.

14 Maia D, de Carvalho AC, Horst MA, Carvalho AL, Scapulatempo-Neto C and Vettore AL: Expression of miR-296$5 p$ as predictive marker for radiotherapy resistance in early-stage laryngeal carcinoma. J Transl Med 13: 262, 2015.

15 Lu Y, Li T, Wei G, Liu L, Chen Q, Xu L, Zhang K, Zeng D and Liao R: The long non-coding RNA NEAT1 regulates epithelial to mesenchymal transition and radioresistance in through miR204/ZEB1 axis in nasopharyngeal carcinoma. Tumour Biol 37: 11733-11741, 2016.

16 Tan J, Qiu K, Li M and Liang Y: Double-negative feedback loop between long non-coding RNA TUG1 and miR-145 promotes epithelial to mesenchymal transition and radioresistance in human bladder cancer cells. FEBS Lett 589: 3175-3181, 2015. 
17 Lu H, He Y, Lin L, Qi Z, Ma L, Li L and Su Y: Long non-coding RNA MALAT1 modulates radiosensitivity of HR-HPV+ cervical cancer via sponging miR-145. Tumour Biol 37: 1683-1691, 2016.

18 Gong P, Zhang T, He D and Hsieh JT: MicroRNA-145 Modulates Tumor Sensitivity to Radiation in Prostate Cancer. Radiat Res 184: 630-638, 2015.

19 Yuan W, Xiaoyun H, Haifeng Q, Jing L, Weixu H, Ruofan D, Jinjin $Y$ and Zongji S: MicroRNA-218 enhances the radiosensitivity of human cervical cancer via promoting radiation induced apoptosis. Int J Med Sci 11: 691-696, 2014.

20 Yang W, Yu H, Shen Y, Liu Y, Yang Z and Sun T: MiR-146b-5p overexpression attenuates stemness and radioresistance of glioma stem cells by targeting HuR/lincRNA-p21/ $\beta$-catenin pathway. Oncotarget 7: 41505-41526, 2016.
21 Liang Z, Ahn J, Guo D, Votaw JR and Shim H: MicroRNA-302 replacement therapy sensitizes breast cancer cells to ionizing radiation. Pharm Res 30: 1008-1016, 2013.

22 Tang Y, Cui Y, Li Z, Jiao Z, Zhang Y, He Y, Chen G, Zhou Q, Wang W, Zhou X, Luo J and Zhang S: Radiation-induced miR208a increases the proliferation and radioresistance by targeting p21 in human lung cancer cells. J Exp Clin Cancer Res 35: 7, 2016.

Received December 27, 2016

Revised January 30, 2017

Accepted January 31, 2017 